\title{
Compactness of semigroups generated by symmetric non-local Dirichlet forms with unbounded coefficients
}

\author{
Yuichi Shiozawa* Jian Wang ${ }^{\dagger}$
}

May 13, 2020

\begin{abstract}
Let $(\mathcal{E}, \mathcal{F})$ be a symmetric non-local Dirichlet from with unbounded coefficient on $L^{2}\left(\mathbb{R}^{d} ; \mathrm{d} x\right)$ defined by

$$
\mathcal{E}(f, g)=\iint_{\mathbb{R}^{d} \times \mathbb{R}^{d}}(f(y)-f(x))(g(x)-g(y)) W(x, y) J(x, \mathrm{~d} y) \mathrm{d} x, \quad f, g \in \mathcal{F},
$$

where $J(x, \mathrm{~d} y)$ is regarded as the jumping kernel for a pure-jump symmetric Lévytype process with bounded coefficients, and $W(x, y)$ is seen as a weighted (unbounded) function. We establish sharp criteria for compactness and non-compactness of the associated Markovian semigroup $\left(P_{t}\right)_{t>0}$ on $L^{2}\left(\mathbb{R}^{d} ; \mathrm{d} x\right)$. In particular, we prove that if $J(x, \mathrm{~d} y)=|x-y|^{-d-\alpha} \mathrm{d} y$ with $\alpha \in(0,2)$, and$$
W(x, y)= \begin{cases}(1+|x|)^{p}+(1+|y|)^{p}, & |x-y|<1 \\ (1+|x|)^{q}+(1+|y|)^{q}, & |x-y| \geq 1\end{cases}
$$

with $p \in[0, \infty)$ and $q \in[0, \alpha)$, then $\left(P_{t}\right)_{t \geq 0}$ is compact, if and only if $p>2$. This indicates that the compactness of $(\mathcal{E}, \overline{\mathcal{F}})$ heavily depends on the growth of the weighted function $W(x, y)$ only for $|x-y|<1$. Our approach is based on establishing the essential super Poincaré inequality for $(\mathcal{E}, \mathcal{F})$. Our general results work even if the jumping kernel $J(x, \mathrm{~d} y)$ is degenerate or is singular with respect to the Lebesgue measure.
\end{abstract}

\section{Introduction}

It is an important research subject in functional analysis and mathematical physics to reveal the spectral structure of a self-adjoint operator $L$ generating a Markovian semigroup

*Department of Mathematics, Graduate School of Science, Osaka University, Toyonaka, Osaka, 5600043, Japan; shiozawa@math.sci.osaka-u.ac.jp

${ }^{\dagger}$ College of Mathematics and Informatics \& Fujian Key Laboratory of Mathematical Analysis and Applications (FJKLMAA) \& Center for Applied Mathematics of Fujian Province (FJNU), Fujian Normal University, Fuzhou, 350007, P.R. China; jianwang@f jnu.edu.cn 
$\left(P_{t}\right)_{t \geq 0}:=\left(e^{-t L}\right)_{t \geq 0}$. In view of the study of symmetric Markov processes, we can deduce asymptotic properties, in particular, ergodic properties, of transition semigroups by using the spectral structure. To do so, we would like to find conditions for compactness of $\left(P_{t}\right)_{t \geq 0}$ because it is equivalent to the essential spectrum of $L$ being empty (see, e.g., [15, Theorem 0.3.9(ii)]). In fact, if this is the case for the symmetric semigroup $\left(P_{t}\right)_{t \geq 0}$ on $L^{2}\left(\mathbb{R}^{d} ; \mathrm{d} x\right)$, then there exist eigenvalues $\left\{\lambda_{n}\right\}_{n \geq 1}$ and the corresponding eigenfunctions $\left\{\varphi_{n}\right\}_{n \geq 1} \subset L^{2}\left(\mathbb{R}^{d} ; \mathrm{d} x\right)$ such that $P_{t} f=\sum_{n=1}^{\infty} e^{-\lambda_{n} t}\left\langle\varphi_{n}, f\right\rangle_{L^{2}\left(\mathbb{R}^{d} ; \mathrm{d} x\right)} \varphi_{n}$ for $f \in L^{2}\left(\mathbb{R}^{d} ; \mathrm{d} x\right)$ and $t>0$. The readers are referred to [17, 12] for several equivalent conditions for the compactness of symmetric Markov semigroups.

If $L$ is a (symmetric) second order elliptic operator of the form $\sum_{i, j=1}^{d} \frac{\partial}{\partial x_{i}}\left(a_{i j}(x) \frac{\partial}{\partial x_{j}}\right)$ on $L^{2}\left(\mathbb{R}^{d} ; \mathrm{d} x\right)$, then the spectral structure of $\left(P_{t}\right)_{t \geq 0}$ is characterized in terms of the growth order of the coefficient $\left\{a_{i j}(x)\right\}_{1 \leq i \leq d}$ at infinity (see, e.g., [3, 4, 8, 9]). More precisely, let $\left\{a_{i j}(x)\right\}_{1 \leq i, j \leq d}$ be a $C^{\infty}$ function on $\mathbb{R}^{d}$ with values in the set of positive symmetric matrices, and $\mathcal{E}^{L}$ the quadratic form on $C_{c}^{\infty}\left(\mathbb{R}^{d}\right)$ defined by

$$
\mathcal{E}^{L}(f, g)=\frac{1}{2} \int_{\mathbb{R}^{d}} \sum_{i, j=1}^{d} a_{i j}(x) \frac{\partial f}{\partial x_{i}}(x) \frac{\partial g}{\partial x_{j}}(x) \mathrm{d} x .
$$

Here $C_{c}^{\infty}\left(\mathbb{R}^{d}\right)$ is the totality of smooth functions on $\mathbb{R}^{d}$ with compact support. For simplicity, we assume that there exist positive constants $\lambda, \Lambda$ and $p$ such that for any $x, \xi \in \mathbb{R}^{d}$,

$$
\lambda(1+|x|)^{p}|\xi|^{2} \leq \sum_{i, j=1}^{d} a_{i j}(x) \xi_{i} \xi_{j} \leq \Lambda(1+|x|)^{p}|\xi|^{2} .
$$

Then, the closure of the quadratic form $\left(\mathcal{E}^{L}, C_{c}^{\infty}\left(\mathbb{R}^{d}\right)\right)$ is a regular Dirichlet form on $L^{2}\left(\mathbb{R}^{d} ; \mathrm{d} x\right)$ generating a Markovian semigroup (see e.g., [5, Chapter 1 and Section 3.1]). In particular, this semigroup is compact, if and only if $p>2$ (see [3, Theorem 4.2 and Corollary 4.4] and Remark 2.2 below).

The purpose of this paper is to establish sharp criteria for compactness and noncompactness of the semigroups associated with a large class of non-local Dirichlet forms on $L^{2}\left(\mathbb{R}^{d} ; \mathrm{d} x\right)$ with unbounded coefficients. To highlight the novelty of our contribution, we present the assertions for stable-like Dirichlet forms with unbounded coefficients in this section, which is a special case of general results (see Theorems 2.1 and 2.4 below).

For $d \geq 1$ and $\alpha \in(0,2)$, the fractional Laplacian is defined by

$$
-(-\Delta)^{\alpha / 2} f(x):=\lim _{\varepsilon \rightarrow 0} \int_{\{|x-y|>\varepsilon\}}(f(y)-f(x)) \frac{C_{d, \alpha}}{|x-y|^{d+\alpha}} \mathrm{d} y,
$$

where $C_{d, \alpha}$ is a positive constant depending on $d$ and $\alpha$. It is known that the essential spectrum of the operator $(-\Delta)^{\alpha / 2}$ is $[0, \infty)$. Equivalently, the associated Markovian semigroup is not compact. On the contrary, this semigroup has nice analytical properties; in particular, it has a strictly positive density function with respect to the Lebesgue measure (see, e.g., [2]). Therefore, according to [15, Theorem 0.3.9 and Theorem 3.2.1], for any strictly positive function $\psi \in L^{2}\left(\mathbb{R}^{d} ; \mathrm{d} x\right)$, there exists no decreasing function 
$\beta:(0, \infty) \rightarrow(0, \infty)$ such that the following essential super Poincaré inequality holds for all $r>0$ and $f \in C_{c}^{\infty}\left(\mathbb{R}^{d}\right)$ :

$$
\int_{\mathbb{R}^{d}} f(x)^{2} \mathrm{~d} x \leq r \mathcal{E}^{0}(f, f)+\beta(r)\left(\int_{\mathbb{R}^{d}}|f(x)| \psi(x) \mathrm{d} x\right)^{2} .
$$

Here $\mathcal{E}^{0}(f, f)$ is the bilinear form associated with the fractional Laplacian $-(-\Delta)^{\alpha / 2}$, i.e., for any $f, g \in C_{c}^{\infty}\left(\mathbb{R}^{d}\right)$,

$\mathcal{E}^{0}(f, g)=\left\langle(-\Delta)^{\alpha / 2} f, g\right\rangle_{L^{2}\left(\mathbb{R}^{d} ; \mathrm{d} x\right)}=\frac{1}{2} \iint_{\mathbb{R}^{d} \times \mathbb{R}^{d}}(f(y)-f(x))(g(x)-g(y)) \frac{C_{d, \alpha}}{|x-y|^{d+\alpha}} \mathrm{d} x \mathrm{~d} y$.

In the theory of stochastic processes, the fractional Laplacian $-(-\Delta)^{\alpha / 2}$ is the infinitesimal generator of the rotationally symmetric $\alpha$-stable process, and so $\mathcal{E}^{0}(f, g)$ is called the $\alpha$-stable Dirichlet form in the literature.

In order for the validity of the inequality (1.3), one reasonable way is to enlarge the bilinear form $\mathcal{E}^{0}(f, f)$ at the right hand side of (1.3); that is, instead of $\mathcal{E}^{0}(f, g)$, we will consider the following $\alpha$-stable like Dirichlet form with unbounded coefficient:

$$
\mathcal{E}(f, g):=\iint_{\mathbb{R}^{d} \times \mathbb{R}^{d}}(f(x)-f(y))(g(x)-g(y)) \frac{W(x, y)}{|x-y|^{d+\alpha}} \mathrm{d} x \mathrm{~d} y,
$$

where $W(x, y)$ is a strictly positive, symmetric and unbounded measurable function on $\mathbb{R}^{d} \times \mathbb{R}^{d}$. We can regard (1.4) as a non-local analogue of (1.1). If (1.3) holds with $\mathcal{E}^{0}(f, f)$ replaced by $\mathcal{E}(f, f)$, then we can immediately get the compactness of the semigroup associated with the bilinear form $\mathcal{E}(f, g)$. This explains the motivation of our paper.

Let $(\mathcal{E}, \mathcal{D}(\mathcal{E}))$ be a quadratic form on $L^{2}\left(\mathbb{R}^{d} ; \mathrm{d} x\right)$ such that $\mathcal{E}$ is as in $(1.4)$, and $\mathcal{D}(\mathcal{E})=$ $\left\{f \in L^{2}\left(\mathbb{R}^{d} ; \mathrm{d} x\right): \mathcal{E}(f, f)<\infty\right\}$. We assume that

$$
x \mapsto \int_{\mathbb{R}^{d}}\left(1 \wedge|x-y|^{2}\right) \frac{W(x, y)}{|x-y|^{d+\alpha}} \mathrm{d} y \in L_{\mathrm{loc}}^{1}\left(\mathbb{R}^{d} ; \mathrm{d} x\right) .
$$

It holds that $C_{c}^{\infty}\left(\mathbb{R}^{d}\right) \subset \mathcal{D}(\mathcal{E})$. Let $\|\cdot\|_{\mathcal{E}_{1}}$ be the norm on $\mathcal{D}(\mathcal{E})$ defined by $\|f\|_{\mathcal{E}_{1}}=$ $\left(\mathcal{E}(f, f)+\|f\|_{L^{2}\left(\mathbb{R}^{d} ; \mathrm{d} x\right)}^{2}\right)^{1 / 2}$, and $\mathcal{F}:=\overline{C_{c}^{\infty}\left(\mathbb{R}^{d}\right)}\|\cdot\|_{\mathcal{E}_{1}}$. Then, $(\mathcal{E}, \mathcal{F})$ is a regular Dirichlet form on $L^{2}\left(\mathbb{R}^{d} ; \mathrm{d} x\right)$. Denote by $\left(P_{t}\right)_{t \geq 0}$ the associated Markovian semigroup. We have the following statement.

Theorem 1.1. Let $W(x, y)$ be a Borel measurable function on $\mathbb{R}^{d} \times \mathbb{R}^{d}$ defined by

$$
W(x, y)=\left(U_{1}(x)+U_{1}(y)\right) \mathbf{1}_{\{|x-y|<1\}}+\left(U_{2}(x)+U_{2}(y)\right) \mathbf{1}_{\{|x-y| \geq 1\}},
$$

where $U_{i}(x)(i=1,2)$ is a nonnegative locally bounded function on $\mathbb{R}^{d}$ such that for some $c_{1}>0$ and $q \in[0, \alpha)$,

$$
U_{2}(x) \leq c_{1}(1+|x|)^{q}, \quad x \in \mathbb{R}^{d} .
$$

Then, we have 
(i) if $\inf _{x \in \mathbb{R}^{d}} U_{1}(x)>0$ and

$$
\liminf _{|x| \rightarrow \infty} \frac{U_{1}(x)}{|x|^{2}}=\infty
$$

then $\left(P_{t}\right)_{t \geq 0}$ is compact on $L^{2}\left(\mathbb{R}^{d} ; \mathrm{d} x\right)$;

(ii) if

$$
\limsup _{|x| \rightarrow \infty} \frac{U_{1}(x)}{|x|^{2}}<\infty,
$$

then $\left(P_{t}\right)_{t \geq 0}$ is not compact on $L^{2}\left(\mathbb{R}^{d} ; \mathrm{d} x\right)$.

(1.7) along with the local boundedness of $U_{i}(i=1,2)$ implies that (1.5) holds. As a direct consequence of Theorem 1.1, by letting

$$
W(x, y)= \begin{cases}(1+|x|)^{p}+(1+|y|)^{p}, & |x-y|<1 \\ (1+|x|)^{q}+(1+|y|)^{q}, & |x-y| \geq 1\end{cases}
$$

with $p \in[0, \infty)$ and $q \in[0, \alpha)$, the associated semigroup $\left(P_{t}\right)_{t \geq 0}$ is compact, if and only if, $p>2$.

We will make a few comments on Theorem 1.1 .

(i) Theorem 1.1 indicates that the compactness of the semigroup associated with the Dirichlet form $(\mathcal{E}, \mathcal{F})$ given by (1.4) heavily depends on the growth of the weighted function $W(x, y)$ only for $|x-y|<1$. Indeed, according to our general results below (see Theorems 2.1 and 2.4), the assertion of Theorem 1.1 still holds true for the truncated version of $(\mathcal{E}, \mathcal{F})$, i.e., the Dirichlet form $\left(\mathcal{E}^{*}, \mathcal{F}^{*}\right)$ on $L^{2}\left(\mathbb{R}^{d} ; \mathrm{d} x\right)$ given by

$$
\mathcal{E}^{*}(f, g):=\iint_{\{|x-y|<1\}}(f(x)-f(y))(g(x)-g(y)) \frac{W(x, y)}{|x-y|^{d+\alpha}} \mathrm{d} x \mathrm{~d} y
$$

and $\mathcal{F}^{*}:=\overline{C_{c}^{\infty}\left(\mathbb{R}^{d}\right)}\|\|_{\mathcal{E}_{1}^{*}}$, where $\|f\|_{\mathcal{E}_{1}^{*}}=\left(\mathcal{E}^{*}(f, f)+\|f\|_{L^{2}\left(\mathbb{R}^{d} ; \mathrm{d} x\right)}^{2}\right)^{1 / 2}$. In particular, for the weighted function $W(x, y)$ given by (1.8), the semigroup associated with $\left(\mathcal{E}^{*}, \mathcal{F}^{*}\right)$ is compact, if and only if, $p>2$. This shows that in this case the criteria for compactness of the semigroup associated with the non-local Dirichlet form $\left(\mathcal{E}^{*}, \mathcal{F}^{*}\right)$ on $L^{2}\left(\mathbb{R}^{d} ; \mathrm{d} x\right)$ with finite range jumping kernel is the same as these for the local Dirichlet form given by (1.1).

(ii) The proof of Theorem 1.1 is based on establishing the essential super Poincaré inequality for $(\mathcal{E}, \mathcal{F})$, which was first introduced by F.-Y. Wang in [14]. However, there are few results concerning the (optimal) essential super Poincaré inequality for non-local Dirichlet forms. In order to prove Theorem 1.1, we will make use of the comparison argument and Hardy-type inequality for $(\mathcal{E}, \mathcal{F}$ ) (see Lemma 2.8). The test function $\phi$ involved in that inequality is given by $\phi(x)=\left(1+|x|^{2}\right)^{-\delta / 2}$ with $\delta \in(0,1)$. Then for any $C>0$, there exist $\lambda>0$ and $R_{0}>0$ such that for any $x \in \mathbb{R}^{d}$ with $|x| \geq R_{0}$,

$$
\frac{-\mathcal{L}_{\lambda} \phi}{\phi}(x) \geq C
$$


where $\left\{\mathcal{L}_{\lambda}\right\}_{\lambda \geq 1}$ is a family of the formal generators of auxiliary quadratic forms dominated by $(\mathcal{E}, \mathcal{F})$; see Propositions 2.5 and 2.6. This approach is powerful in the sense that we can even deduce the corresponding statements for more general non-local Dirichlet forms with unbounded coefficients.

(iii) Recently, the compactness of the weighted fractional heat semigroups has been studied. Let $\check{\mathcal{L}}=-(1+|x|)^{p}(-\Delta)^{\alpha / 2}$ be a (formal) self-adjoint operator on $L^{2}\left(\mathbb{R}^{d} ; \mathrm{d} x /(1+\right.$ $|x|)^{p}$ ) with $p \geq 0$ and $d>\alpha$. Then, the associated Markovian semigroup is compact, if and only if $p>\alpha$; see [13, Proposition 4.1] and [16, Corollary 2.3]. See also [7, Theorems 4.8 and 2.2] for the study of compactness for the semigroup on $L^{1}\left(\mathbb{R}^{d} ; \mathrm{d} x /(1+|x|)^{p}\right)$. In fact, with the aid of the Hardy inequality or the fractional Sobolev inequality, the second named author ([16]) proved that the following essential super Poincaré inequality

$$
\int_{\mathbb{R}^{d}} f(x)^{2} \frac{1}{(1+|x|)^{p}} \mathrm{~d} x \leq r \mathcal{E}^{0}(f, f)+\beta(r)\left(\int_{\mathbb{R}^{d}}|f(x)| \frac{\psi(x)}{(1+|x|)^{p}} \mathrm{~d} x\right)^{2}
$$

holds for all $r>0$ and $f \in C_{c}^{\infty}\left(\mathbb{R}^{d}\right)$ with some deceasing function $\beta:(0, \infty) \rightarrow$ $(0, \infty)$ and some function $\psi \in L^{2}\left(\mathbb{R}^{d} ; \mathrm{d} x\right)$, if and only if, $p>\alpha$; see the proof of [16, Theorem 1.2] for more details. Thus, one may try to replace the function $f(x)$ by $f(x)(1+|x|)^{p / 2}$ in (1.9), and then to establish the desired essential super Poincaré inequality for the Dirichlet form $(\mathcal{E}, \mathcal{F})$ given by (1.4). However, it seems that this approach does not work, and is far from getting the sharp result as shown in Theorem 1.1.

The rest of the paper is arranged as follows. In Section 2, we present assertions and their proofs for the compactness and non-compactness criteria of semigroups associated with general non-local Dirichlet forms with unbounded coefficients; see Theorems 2.1 and 2.4. As mentioned above, main tasks of the proofs are to disprove and to establish essential super Poincaré inequalities for the associated Dirichlet form. In Section 3 , we present the proof of Theorem 1.1, and also give two examples of non-local Dirichlet forms with degenerate or singular jumping kernel to illustrate our general results.

\section{General results}

Let $J(x, \mathrm{~d} y)$ be a nonnegative kernel on $\mathbb{R}^{d} \times \mathcal{B}\left(\mathbb{R}^{d}\right)$ such that the measure $J(\mathrm{~d} x, \mathrm{~d} y):=$ $J(x, \mathrm{~d} y) \mathrm{d} x$ satisfies the symmetry condition

$$
J(\mathrm{~d} x, \mathrm{~d} y)=J(\mathrm{~d} y, \mathrm{~d} x),
$$

and

$$
\sup _{x \in \mathbb{R}^{d}} \int_{\mathbb{R}^{d}}\left(1 \wedge|x-y|^{2}\right) J(x, \mathrm{~d} y)<\infty
$$

That is, $J(x, \mathrm{~d} y)$ is regarded as the jumping kernel for a pure-jump Lévy-type process with bounded coefficients. Let $W(x, y)$ be a nonnegative, locally bounded and symmetric 
Borel measurable function on $\mathbb{R}^{d} \times \mathbb{R}^{d}$ such that

$$
x \mapsto \int_{\mathbb{R}^{d}}\left(1 \wedge|x-y|^{2}\right) W(x, y) J(x, \mathrm{~d} y) \in L_{\mathrm{loc}}^{1}\left(\mathbb{R}^{d} ; \mathrm{d} x\right) .
$$

Roughly speaking, $W(x, y)$ is regarded as a weighted function.

Let $(\mathcal{E}, \mathcal{D}(\mathcal{E}))$ be a quadratic form on $L^{2}\left(\mathbb{R}^{d} ; \mathrm{d} x\right)$ defined by

$$
\begin{aligned}
\mathcal{D}(\mathcal{E}) & =\left\{u \in L^{2}\left(\mathbb{R}^{d} ; \mathrm{d} x\right): \iint_{\mathbb{R}^{d} \times \mathbb{R}^{d}}(u(x)-u(y))^{2} W(x, y) J(x, \mathrm{~d} y) \mathrm{d} x<\infty\right\}, \\
\mathcal{E}(u, u) & =\iint_{\mathbb{R}^{d} \times \mathbb{R}^{d}}(u(x)-u(y))^{2} W(x, y) J(x, \mathrm{~d} y) \mathrm{d} x .
\end{aligned}
$$

It is easy to verify that $C_{c}^{\infty}\left(\mathbb{R}^{d}\right) \subset \mathcal{D}(\mathcal{E})$ under $(2.3)$. Define the norm $\|\cdot\|_{\mathcal{E}_{1}}$ on $\mathcal{D}(\mathcal{E})$ by

$$
\|f\|_{\mathcal{E}_{1}}=\left(\mathcal{E}(f, f)+\|f\|_{L^{2}\left(\mathbb{R}^{d} ; \mathrm{d} x\right)}^{2}\right)^{1 / 2} .
$$

We can then define

$$
\mathcal{F}:=\overline{C_{c}^{\infty}\left(\mathbb{R}^{d}\right)}\|\cdot\|_{\mathcal{E}_{1}},
$$

so that $(\mathcal{E}, \mathcal{F})$ is a regular Dirichlet form on $L^{2}\left(\mathbb{R}^{d} ; \mathrm{d} x\right)$, see, e.g., [5, Example 1.2.4]. We denote by $\left(P_{t}\right)_{t \geq 0}$ the $L^{2}$-semigroup associated with $(\mathcal{E}, \mathcal{F})$.

\subsection{Condition for non-compactness}

In this subsection, we provide a sufficient condition for non-compactness of $\left(P_{t}\right)_{t \geq 0}$.

Theorem 2.1. Suppose that

$$
\liminf _{l \rightarrow \infty}\left[l^{-d} \int_{\{|x| \leq l\}} \int_{\mathbb{R}^{d}}\left(1 \wedge \frac{|x-y|^{2}}{l^{2}}\right) W(x, y) J(x, \mathrm{~d} y) \mathrm{d} x\right]<\infty .
$$

Then, the semigroup $\left(P_{t}\right)_{t \geq 0}$ is not compact on $L^{2}\left(\mathbb{R}^{d} ; \mathrm{d} x\right)$.

Proof. We apply some idea in the proof of [16, Theorem 2.2(ii)]. Suppose that $\left(P_{t}\right)_{t \geq 0}$ is compact on $L^{2}\left(\mathbb{R}^{d} ; \mathrm{d} x\right)$. We then see by [15, Theorem 0.3.9 and Theorem 3.2.1] that for any strictly positive function $\psi \in L^{2}\left(\mathbb{R}^{d} ; \mathrm{d} x\right)$, there exists a strictly positive nondecreasing function $\beta(r)$ on $(0, \infty)$ such that for any $r>0$ and $f \in C_{c}^{\infty}\left(\mathbb{R}^{d}\right)$,

$$
\int_{\mathbb{R}^{d}} f(x)^{2} \mathrm{~d} x \leq r \mathcal{E}(f, f)+\beta(r)\left(\int_{\mathbb{R}^{d}}|f(x)| \psi(x) \mathrm{d} x\right)^{2} .
$$

We now take $\psi(x)=e^{-|x|}$. For $l \geq 1$, let $f_{l} \in C_{c}^{\infty}\left(\mathbb{R}^{d}\right)$ satisfy

$$
f_{l}(x) \begin{cases}=1, & 0 \leq|x| \leq l \\ \in[0,1], & l \leq|x| \leq 2 l \\ =0, & |x| \geq 2 l\end{cases}
$$


and $\left\|\nabla f_{l}\right\|_{\infty} \leq 2 l^{-1}$. Then

$$
\int_{\mathbb{R}^{d}} f_{l}(x)^{2} \mathrm{~d} x \geq c_{1} l^{d}
$$

and

$$
\int_{\mathbb{R}^{d}}\left|f_{l}(x)\right| \psi(x) \mathrm{d} x \leq \int_{\mathbb{R}^{d}} \psi(x) \mathrm{d} x=c_{2} .
$$

Here and in what follows, all the constants $c_{i}$ are independent of $l$. Furthermore,

$$
\begin{aligned}
\mathcal{E}\left(f_{l}, f_{l}\right)= & \iint_{\mathbb{R}^{d} \times \mathbb{R}^{d}}\left(f_{l}(x)-f_{l}(y)\right)^{2} W(x, y) J(x, \mathrm{~d} y) \mathrm{d} x \\
= & 2 \int_{\{|x| \leq 2 l\}} \int_{\mathbb{R}^{d}}\left(f_{l}(x)-f_{l}(y)\right)^{2} W(x, y) J(x, \mathrm{~d} y) \mathrm{d} x \\
= & 2 \int_{\{|x| \leq 2 l\}} \int_{\{|y|<4 l\}}\left(f_{l}(x)-f_{l}(y)\right)^{2} W(x, y) J(x, \mathrm{~d} y) \mathrm{d} x \\
& +2 \int_{\{|x| \leq 2 l\}} \int_{\{|y| \geq 4 l\}}\left(f_{l}(x)-f_{l}(y)\right)^{2} W(x, y) J(x, \mathrm{~d} y) \mathrm{d} x \\
= & : 2(\mathrm{I})+2(\mathrm{II}),
\end{aligned}
$$

where in the second equality we used the fact that $\operatorname{supp}\left(f_{l}\right) \subset B(0,2 l)$, (2.1) and the symmetry of $W(x, y)$. Here and in what follows, $B(0, r):=\left\{x \in \mathbb{R}^{d}:|x|<r\right\}$ for $r>0$.

We have

$$
\begin{aligned}
(\mathrm{I}) & \leq \int_{\{|x| \leq 2 l\}} \int_{\{|x-y|<6 l\}}\left(f_{l}(x)-f_{l}(y)\right)^{2} W(x, y) J(x, \mathrm{~d} y) \mathrm{d} x \\
& \leq 4 l^{-2} \int_{\{|x| \leq 2 l\}} \int_{\{|x-y|<6 l\}}|x-y|^{2} W(x, y) J(x, \mathrm{~d} y) \mathrm{d} x
\end{aligned}
$$

and

$$
\begin{aligned}
(\mathrm{II}) \leq & \int_{\{|x| \leq 2 l\}} \int_{\{|x-y| \geq 2 l\}} W(x, y) J(x, \mathrm{~d} y) \mathrm{d} x \\
\leq & 4^{-1} l^{-2} \int_{\{|x| \leq 2 l\}} \int_{\{2 l \leq|x-y|<6 l\}}|x-y|^{2} W(x, y) J(x, \mathrm{~d} y) \mathrm{d} x \\
& +\int_{\{|x| \leq 2 l\}} \int_{\{|x-y| \geq 6 l\}} W(x, y) J(x, \mathrm{~d} y) \mathrm{d} x .
\end{aligned}
$$

Hence,

$$
\mathcal{E}\left(f_{l}, f_{l}\right) \leq c_{3} \int_{\{|x| \leq 6 l\}} \int_{\mathbb{R}^{d}}\left(1 \wedge \frac{|x-y|^{2}}{(6 l)^{2}}\right) W(x, y) J(x, \mathrm{~d} y) \mathrm{d} x .
$$

Putting (2.6), (2.7) and (2.8) into (2.5) with $f=f_{l}$, we find that there are constants $c_{4}, c_{5}>0$ such that for all $l \geq 1$ and $r>0$.

$$
l^{d} \leq c_{4} r \int_{\{|x| \leq 6 l\}} \int_{\mathbb{R}^{d}}\left(1 \wedge \frac{|x-y|^{2}}{(6 l)^{2}}\right) W(x, y) J(x, \mathrm{~d} y) \mathrm{d} x+c_{5} \beta(r) ;
$$


that is,

$$
1 \leq c_{4} r\left[l^{-d} \int_{\{|x| \leq 6 l\}} \int_{\mathbb{R}^{d}}\left(1 \wedge \frac{|x-y|^{2}}{(6 l)^{2}}\right) W(x, y) J(x, \mathrm{~d} y) \mathrm{d} x\right]+c_{5} \beta(r) l^{-d} .
$$

However, under (2.4), we have a contradiction from the inequality above by taking liminf for $l \rightarrow \infty$ and then letting $r \rightarrow 0$. Namely, $\left(P_{t}\right)_{t \geq 0}$ is not compact on $L^{2}\left(\mathbb{R}^{d} ; \mathrm{d} x\right)$.

Remark 2.2. Let $\left(\mathcal{E}^{L}, C_{c}^{\infty}\left(\mathbb{R}^{d}\right)\right)$ be a quadratic form as in (1.1). Suppose that $\left\{a_{i j}(x)\right\}_{1 \leq i, j \leq d}$ is local uniformly elliptic, and that there exists a positive Borel measurable function $a(x)$ on $\mathbb{R}^{d}$ such that for any $x, \xi \in \mathbb{R}^{d}$,

$$
\sum_{i, j=1}^{d} a_{i j}(x) \xi_{i} \xi_{j} \leq a(x)|\xi|^{2}
$$

Denote by $\mathcal{F}^{L}$ the closure of $C_{c}^{\infty}\left(\mathbb{R}^{d}\right)$, i.e., $\mathcal{F}^{L}:={\overline{C_{c}^{\infty}\left(\mathbb{R}^{d}\right)}}^{\|\cdot\|_{\mathcal{E}_{1}^{L}}}$ with $\|f\|_{\mathcal{E}_{1}^{L}}=\left(\mathcal{E}^{L}(f, f)+\right.$ $\left.\|f\|_{L^{2}\left(\mathbb{R}^{d} ; \mathrm{d} x\right)}^{2}\right)^{1 / 2}$. By analogy with the proof of Theorem 2.1, we can show that the semigroup associated with $\left(\mathcal{E}^{L}, \mathcal{F}^{L}\right)$ is non-compact, if

$$
\liminf _{l \rightarrow \infty}\left[l^{-(d+2)} \int_{\{|x| \leq 2 l\}} a(x) \mathrm{d} x\right]<\infty .
$$

In particular, if $\left\{a_{i j}(x)\right\}_{1 \leq i, j \leq d}$ satisfies (1.2), then the semigroup associated with $\left(\mathcal{E}^{L}, \mathcal{F}^{L}\right)$ is non-compact when $p \leq 2$.

\subsection{Condition for compactness}

We first recall that $W(x, y)$ and $J(x, \mathrm{~d} y)$ satisfy (2.3). To establish a sufficient condition for compactness of the semigroup $\left(P_{t}\right)_{t \geq 0}$, we will impose the following conditions on $W(x, y)$ and $J(x, \mathrm{~d} y)$, respectively.

Assumption 2.3. (i) The function $W(x, y)$ is locally bounded on $\mathbb{R}^{d} \times \mathbb{R}^{d}$ such that for any $\lambda>0$, there exist a strictly positive $C^{1}$-function $V_{\lambda}(x)$ on $\mathbb{R}^{d}$ and a constant $R_{0}>0$ so that

- $W(x, y) \geq V_{\lambda}(x)+V_{\lambda}(y)$ for any $x, y \in \mathbb{R}^{d}$ with $|x-y|<1$;

- $\inf _{x \in \mathbb{R}^{d}} V_{\lambda}(x)>0$, and $V_{\lambda}(x)=\lambda\left(1+|x|^{2}\right)$ for any $x \in B\left(0, R_{0}\right)^{c}$.

(ii) There exists a nonnegative Borel measure $\nu(\mathrm{d} z)$ on $[0,1)$ such that

- $J(x, x+A) \geq \nu(A)$ for any $x \in \mathbb{R}^{d}$ and Borel set $A \subset B(0,1)$;

- $\nu(A)=\nu(-A)$ for any Borel set $A \subset B(0,1)$, where $-A=\left\{x \in \mathbb{R}^{d} \mid-x \in A\right\}$; 
- Let

$$
\varphi(\xi)=\int_{\{|z|<1\}}(1-\cos \langle z, \xi\rangle) \nu(\mathrm{d} z), \quad \xi \in \mathbb{R}^{d}
$$

and

$$
\beta_{0}(r)=\int_{\mathbb{R}^{d}} e^{-r \varphi(\xi)} \mathrm{d} \xi, \quad r>0
$$

Then

$$
\beta_{0}(r)<\infty, \quad \int_{r}^{\infty} \frac{\beta_{0}^{-1}(s)}{s} \mathrm{~d} s<\infty, \quad r>0
$$

where $\beta_{0}^{-1}(r)$ is an inverse function of $\beta_{0}(r)$.

Under Assumption 2.3(i), we have

$$
\inf _{x, y \in \mathbb{R}^{d:|x-y|<1}} W(x, y)>0 .
$$

Note also that by (2.2), the measure $\nu$ in Assumption 2.3)(ii) satisfies

$$
\int_{\{0<|z|<1\}}|z|^{2} \nu(\mathrm{d} z) \leq \sup _{x \in \mathbb{R}^{d}} \int_{\{0<|x-y|<1\}}|x-y|^{2} J(x, y) \mathrm{d} y<\infty .
$$

We then have

Theorem 2.4. Under Assumption 2.3 , the semigroup $\left(P_{t}\right)_{t \geq 0}$ is compact on $L^{2}\left(\mathbb{R}^{d} ; \mathrm{d} x\right)$.

To prove Theorem 2.4, we need to introduce a class of auxiliary quadratic forms. For any fixed $\lambda>0$, we define $W_{\lambda}(x, y)=V_{\lambda}(x)+V_{\lambda}(y)$, and

$$
\mathcal{E}^{\lambda}(u, u)=\iint_{\{0<|z|<1\}}(u(x+z)-u(x))^{2} W_{\lambda}(x, x+z) \nu(\mathrm{d} z) \mathrm{d} x, \quad u \in \mathcal{F} .
$$

Then by Assumption 2.3 , $\mathcal{E}^{\lambda}(u, u) \leq \mathcal{E}(u, u)$ for any $u \in \mathcal{F}$.

For our purpose, we will extend the domain of the quadratic form $\left(\mathcal{E}^{\lambda}, \mathcal{F}\right)$. For a pair $f$ and $g$ of Borel measurable functions on $\mathbb{R}^{d}$ such that

$$
\iint_{\{0<|z|<1\}}|f(x+z)-f(x)||g(x+z)-g(x)| W_{\lambda}(x, x+z) \nu(\mathrm{d} z) \mathrm{d} x<\infty,
$$

we let

$$
\mathcal{E}^{\lambda}(f, g):=\iint_{\{0<|z|<1\}}(f(x+z)-f(x))(g(x+z)-g(x)) W_{\lambda}(x, x+z) \nu(\mathrm{d} z) \mathrm{d} x .
$$

It is clear that $\mathcal{E}^{\lambda}(f, g)$ is well defined, if $f, g \in \mathcal{F}$ (in particular, if $f, g \in C_{c}^{\infty}\left(\mathbb{R}^{d}\right)$ ).

Proposition 2.5. Let $\phi(x)=\left(1+|x|^{2}\right)^{-\delta / 2}$ for some $\delta>0$. Under Assumption 2.3 , we have the following statements for any $\lambda>0$. 
(i) For any $g \in C_{c}^{\infty}\left(\mathbb{R}^{d}\right)$,

$$
\iint_{\{0<|z|<1\}}|\phi(x+z)-\phi(x)||g(x+z)-g(x)| W_{\lambda}(x, x+z) \nu(\mathrm{d} z) \mathrm{d} x<\infty .
$$

In particular, $\mathcal{E}^{\lambda}(\phi, g)$ is well defined.

(ii) For any $g \in C_{c}^{\infty}\left(\mathbb{R}^{d}\right)$,

$$
\iint_{\{0<|z|<1\}}|\phi(x+z)-\phi(x)-\langle\nabla \phi(x), z\rangle||g(x)| W_{\lambda}(x, x+z) \nu(\mathrm{d} z) \mathrm{d} x<\infty
$$

and

$$
\iint_{\{0<|z|<1\}}|\langle\nabla \phi(x), z\rangle||g(x)|\left|W_{\lambda}(x, x+z)-W_{\lambda}(x, x-z)\right| \nu(\mathrm{d} z) \mathrm{d} x<\infty .
$$

(iii) For any $g \in C_{c}^{\infty}\left(\mathbb{R}^{d}\right)$,

$$
\mathcal{E}^{\lambda}(\phi, g)=-2 \int_{\mathbb{R}^{d}}\left(\mathcal{L}_{\lambda} \phi\right)(x) g(x) \mathrm{d} x,
$$

where

$$
\begin{aligned}
\mathcal{L}_{\lambda} \phi(x)= & \int_{\{0<|z|<1\}}(\phi(x+z)-\phi(x)-\langle\nabla \phi(x), z\rangle) W_{\lambda}(x, x+z) \nu(\mathrm{d} z) \\
& +\frac{1}{2} \int_{\{0<|z|<1\}}\langle\nabla \phi(x), z\rangle\left(W_{\lambda}(x, x+z)-W_{\lambda}(x, x-z)\right) \nu(\mathrm{d} z) .
\end{aligned}
$$

In particular, $\mathcal{L}_{\lambda} \phi$ is locally bounded on $\mathbb{R}^{d}$.

Proof. We first prove (i). Let $g \in C_{c}^{\infty}\left(\mathbb{R}^{d}\right)$. Since $g$ has compact support, there exists a compact set $K \subset \mathbb{R}^{d}$ such that

$$
\begin{aligned}
& \iint_{\{0<|z|<1\}}|\phi(x+z)-\phi(x)||g(x+z)-g(x)| W_{\lambda}(x, x+z) \nu(\mathrm{d} z) \mathrm{d} x \\
& =\int_{K} \int_{\{0<|z|<1\}}|\phi(x+z)-\phi(x)||g(x+z)-g(x)| W_{\lambda}(x, x+z) \nu(\mathrm{d} z) \mathrm{d} x .
\end{aligned}
$$

By the mean value theorem, there exists $c_{1}>0$ such that for any $x, z \in \mathbb{R}^{d}$ with $|z|<1$,

$$
|\phi(x+z)-\phi(x)| \leq \frac{c_{1}|z|}{\left(1+|x|^{2}\right)^{(\delta+1) / 2}} \leq c_{1}|z| .
$$

Then, according to (2.9) and the local boundedness of $W_{\lambda}(x, y)$,

$$
\int_{K} \int_{\{0<|z|<1\}}(\phi(x+z)-\phi(x))^{2} W_{\lambda}(x, x+z) \nu(\mathrm{d} z) \mathrm{d} x
$$




$$
\leq c_{2} \int_{K} \int_{\{0<|z|<1\}}|z|^{2} W_{\lambda}(x, x+z) \nu(\mathrm{d} z) \mathrm{d} x<\infty .
$$

Hence by the Cauchy-Schwarz inequality,

$$
\begin{aligned}
& {\left[\int_{K} \int_{\{0<|z|<1\}}|\phi(x+z)-\phi(x)||g(x+z)-g(x)| W_{\lambda}(x, x+z) \nu(\mathrm{d} z) \mathrm{d} x\right]^{2}} \\
& \leq \int_{K} \int_{\{0<|z|<1\}}(\phi(x+z)-\phi(x))^{2} W_{\lambda}(x, x+z) \nu(\mathrm{d} z) \mathrm{d} x \\
& \quad \times \int_{K} \int_{\{0<|z|<1\}}(g(x+z)-g(x))^{2} W_{\lambda}(x, x+z) \nu(\mathrm{d} z) \mathrm{d} x<\infty,
\end{aligned}
$$

which implies (i).

We next prove (ii) and (iii) in a similar way to the proof of [11, Theorem 2.2]. Fix $\varepsilon>0$. Then, by the symmetry of $\nu(\mathrm{d} z)$ and $W_{\lambda}(x, y)$,

$$
\begin{aligned}
& \iint_{\{\varepsilon<|z|<1\}}(\phi(x+z)-\phi(x))(g(x+z)-g(x)) W_{\lambda}(x, x+z) \nu(\mathrm{d} z) \mathrm{d} x \\
& =-2 \iint_{\{\varepsilon<|z|<1\}}(\phi(x+z)-\phi(x)) g(x) W_{\lambda}(x, x+z) \nu(\mathrm{d} z) \mathrm{d} x \\
& =-2 \iint_{\{\varepsilon<|z|<1\}}(\phi(x+z)-\phi(x)-\langle\nabla \phi(x), z\rangle) g(x) W_{\lambda}(x, x+z) \nu(\mathrm{d} z) \mathrm{d} x \\
& \quad-2 \iint_{\{\varepsilon<|z|<1\}}\langle\nabla \phi(x), z\rangle g(x) W_{\lambda}(x, x+z) \nu(\mathrm{d} z) \mathrm{d} x
\end{aligned}
$$

By the Taylor theorem, there exists $c_{3}>0$ such that for any $x, z \in \mathbb{R}^{d}$ with $|z|<1$,

$$
|\phi(x+z)-\phi(x)-\langle\nabla \phi(x), z\rangle| \leq \frac{c_{3}|z|^{2}}{\left(1+|x|^{2}\right)^{(\delta+2) / 2}} \leq c_{3}|z|^{2},
$$

which implies that

$$
\begin{aligned}
& \int_{\{\varepsilon<|z|<1\}}|\phi(x+z)-\phi(x)-\langle\nabla \phi(x), z\rangle| W_{\lambda}(x, x+z) \nu(\mathrm{d} z) \\
& \leq c_{3} \int_{\{0<|z|<1\}}|z|^{2} W_{\lambda}(x, x+z) \nu(\mathrm{d} z)=: M_{1}(x) .
\end{aligned}
$$

Note that, by (2.9) and the local boundedness of $W_{\lambda}(x, y)$ again, the function $M_{1}(x)$ is locally bounded on $\mathbb{R}^{d}$. Since $g$ has compact support in $\mathbb{R}^{d}$, we have

$$
\begin{aligned}
& \iint_{\{\varepsilon<|z|<1\}}|\phi(x+z)-\phi(x)-\langle\nabla \phi(x), z\rangle||g(x)| W_{\lambda}(x, x+z) \nu(\mathrm{d} z) \mathrm{d} x \\
& \leq \int_{\mathbb{R}^{d}} M_{1}(x)|g(x)| \mathrm{d} x<\infty
\end{aligned}
$$


Since the measure $\nu(\mathrm{d} z)$ is symmetric, we have

$$
\begin{aligned}
& \iint_{\{\varepsilon<|z|<1\}}\langle\nabla \phi(x), z\rangle g(x) W_{\lambda}(x, x+z) \nu(\mathrm{d} z) \mathrm{d} x \\
& =\frac{1}{2} \iint_{\{\varepsilon<|z|<1\}}\langle\nabla \phi(x), z\rangle g(x)\left(W_{\lambda}(x, x+z)-W_{\lambda}(x, x-z)\right) \nu(\mathrm{d} z) \mathrm{d} x .
\end{aligned}
$$

By the mean value theorem and $V_{\lambda} \in C^{1}\left(\mathbb{R}^{d}\right)$, there exists a locally bounded nonnegative function $c_{\lambda}(x, z)$ on $\mathbb{R}^{d} \times \mathbb{R}^{d}$ such that for any $x, z \in \mathbb{R}^{d}$ with $|z|<1$,

$$
\left|W_{\lambda}(x, x+z)-W_{\lambda}(x, x-z)\right|=\left|V_{\lambda}(x+z)-V_{\lambda}(x-z)\right| \leq c_{\lambda}(x, z)|z|,
$$

which implies that

$$
\begin{aligned}
& \int_{\{\varepsilon<|z|<1\}}|\langle\nabla \phi(x), z\rangle|\left|W_{\lambda}(x, x+z)-W_{\lambda}(x, x-z)\right| \nu(\mathrm{d} z) \\
& \leq c_{4} \int_{\{0<|z|<1\}}|z|^{2} c_{\lambda}(x, z) \nu(\mathrm{d} z)=: M_{2}(x) .
\end{aligned}
$$

Then, thanks to (2.9) again, the function $M_{2}(x)$ is also locally bounded on $\mathbb{R}^{d}$. In particular,

$$
\begin{aligned}
& \iint_{\{\varepsilon<|z|<1\}}|\langle\nabla \phi(x), z\rangle||g(x)|\left|W_{\lambda}(x, x+z)-W_{\lambda}(x, x-z)\right| \nu(\mathrm{d} z) \mathrm{d} x \\
& \leq \int_{\mathbb{R}^{d}} M_{2}(x)|g(x)| \mathrm{d} x<\infty .
\end{aligned}
$$

We thus have the assertion (ii) by letting $\varepsilon \rightarrow 0$ in (2.12) and (2.14). We also obtain the assertion (iii) by (2.11), (2.13) and (ii).

Proposition 2.6. For any $\delta \in(0,1)$ and $C>0$, there exist $\lambda>0$ and $R_{0}>0$ such that for any $x \in B\left(0, R_{0}\right)^{c}$, the function $\phi(x)=\left(1+|x|^{2}\right)^{-\delta / 2}$ satisfies

$$
\frac{-\mathcal{L}_{\lambda} \phi}{\phi}(x) \geq C \text {. }
$$

In particular, the function $-\mathcal{L}_{\lambda} \phi / \phi$ is bounded from below.

Proof. According to the proof of Proposition 2.5, we know that $\mathcal{L}_{\lambda} \phi$ is locally bounded. So it suffices to prove (2.15).

Let $\delta>0$. Then for any $x, z \in \mathbb{R}^{d}$ with $0<|z|<1$, we have by the Taylor theorem,

$$
\phi(x+z)-\phi(x)-\langle\nabla \phi(x), z\rangle=\delta \int_{0}^{1}(1-s) \frac{(\delta+2)\langle x+s z, z\rangle^{2}-\left(1+|x+s z|^{2}\right)|z|^{2}}{\left(1+|x+s z|^{2}\right)^{\delta / 2+2}} \mathrm{~d} s .
$$

Then, the Fubuni theorem yields that for any $\lambda>0$,

$$
\int_{\{0<|z|<1\}}(\phi(x+z)-\phi(x)-\langle\nabla \phi(x), z\rangle) W_{\lambda}(x, x+z) \nu(\mathrm{d} z)
$$




$$
\begin{aligned}
= & \delta \int_{0}^{1}(1-s) \int_{\{0<|z|<1\}} \frac{(\delta+2)\langle x+s z, z\rangle^{2}-\left(1+|x+s z|^{2}\right)|z|^{2}}{\left(1+|x+s z|^{2}\right)^{\delta / 2+2}} W_{\lambda}(x, x+z) \nu(\mathrm{d} z) \mathrm{d} s \\
= & \delta(\delta+2) \int_{0}^{1}(1-s) \int_{\{0<|z|<1\}} \frac{\langle x+s z, z\rangle^{2}}{\left(1+|x+s z|^{2}\right)^{\delta / 2+2}} W_{\lambda}(x, x+z) \nu(\mathrm{d} z) \mathrm{d} s \\
& -\delta \int_{0}^{1}(1-s) \int_{\{0<|z|<1\}} \frac{|z|^{2}}{\left(1+|x+s z|^{2}\right)^{\delta / 2+1}} W_{\lambda}(x, x+z) \nu(\mathrm{d} z) \mathrm{d} s \\
= & :(\mathrm{I})-(\mathrm{II}) .
\end{aligned}
$$

For any $\delta>0, \varepsilon \in(0,1)$ and $\lambda>0$, there exists a constant $R_{0}>0$ by Assumption 2.3 (i) such that for any $x \in B\left(0, R_{0}\right)^{c}$ and $z \in \mathbb{R}^{d}$ with $0<|z|<1$,

$$
W_{\lambda}(x, x \pm z)=\lambda\left(1+|x|^{2}\right)+\lambda\left(1+|x \pm z|^{2}\right)
$$

in particular, for any $x \in B\left(0, R_{0}\right)^{c}, z \in \mathbb{R}^{d}$ with $0<|z|<1$, and $s \in(0,1)$,

$$
\frac{W_{\lambda}(x, x+z)}{\left(1+|x+s z|^{2}\right)^{\delta / 2+2}} \leq \frac{2 \lambda(1+\varepsilon)}{\left(1+|x|^{2}\right)^{\delta / 2+1}}
$$

and

$$
\frac{W_{\lambda}(x, x+z)}{\left(1+|x+s z|^{2}\right)^{\delta / 2+1}} \geq \frac{2 \lambda(1-\varepsilon)}{\left(1+|x|^{2}\right)^{\delta / 2}}=2 \lambda(1-\varepsilon) \phi(x) .
$$

Then, by (2.18),

$$
(\mathrm{I}) \leq \delta(\delta+2) \frac{2 \lambda(1+\varepsilon)}{\left(1+|x|^{2}\right)^{\delta / 2+1}} \int_{0}^{1}(1-s)\left(\int_{\{0<|z|<1\}}\langle x+s z, z\rangle^{2} \nu(\mathrm{d} z)\right) \mathrm{d} s=:(\mathrm{I})_{*} .
$$

By the symmetry of the measure $\nu(\mathrm{d} z)$,

$$
\int_{\{0<|z|<1\}}\langle x, z\rangle|z|^{2} \nu(\mathrm{d} z)=0 .
$$

Since

$$
\langle x+s z, z\rangle^{2}=\left(\langle x, z\rangle+s|z|^{2}\right)^{2}=\langle x, z\rangle^{2}+2 s\langle x, z\rangle|z|^{2}+s^{2}|z|^{4}
$$

we have

$$
\int_{\{0<|z|<1\}}\langle x+s z, z\rangle^{2} \nu(\mathrm{d} z)=F(x)|x|^{2}+c_{1} s^{2},
$$

where

$$
F(x)=\frac{1}{|x|^{2}} \int_{\{0<|z|<1\}}\langle x, z\rangle^{2} \nu(\mathrm{d} z), \quad c_{1}=\int_{\{0<|z|<1\}}|z|^{4} \nu(\mathrm{d} z) .
$$

Therefore, for any $s \in(0,1)$ and $x \in \mathbb{R}^{d}$,

$$
\int_{0}^{1}(1-s)\left(\int_{\{0<|z|<1\}}\langle x+s z, z\rangle^{2} \nu(\mathrm{d} z)\right) \mathrm{d} s=\frac{F(x)}{2}|x|^{2}+\frac{c_{1}}{12} .
$$


Then, by (2.20), we obtain for any $x \in B_{0}\left(0, R_{0}\right)^{c}$,

$$
\begin{aligned}
(\mathrm{I}) \leq(\mathrm{I})_{*} & \leq \delta(\delta+2) \frac{2 \lambda(1+\varepsilon)}{\left(1+|x|^{2}\right)^{\delta / 2+1}}\left(\frac{F(x)}{2}|x|^{2}+\frac{c_{1}}{12}\right) \\
& \leq \lambda(1+\varepsilon) \delta(\delta+2)\left(F(x)+\frac{c_{1}}{6|x|^{2}}\right) \phi(x) .
\end{aligned}
$$

Let

$$
\gamma_{0}=\int_{\{0<|z|<1\}}|z|^{2} \nu(\mathrm{d} z) .
$$

Then, by (2.19), we have for any $x \in B\left(0, R_{0}\right)^{c}$,

$$
\text { (II) } \geq 2 \lambda \delta(1-\varepsilon) \phi(x) \int_{0}^{1}(1-s) \mathrm{d} s\left(\int_{\{0<|z|<1\}}|z|^{2} \nu(\mathrm{d} z)\right)=\lambda \delta \gamma_{0}(1-\varepsilon) \phi(x) .
$$

Combining this with (2.21), we get that for any $x \in B\left(0, R_{0}\right)^{c}$,

$$
\begin{aligned}
& \int_{\{0<|z|<1\}}(\phi(x+z)-\phi(x)-\langle\nabla \phi(x), z\rangle) W_{\lambda}(x, x+z) \nu(\mathrm{d} z) \\
& =(\mathrm{I})-(\mathrm{II}) \\
& \leq \lambda \delta\left\{(1+\varepsilon)(\delta+2)\left(F(x)+\frac{c_{1}}{6|x|^{2}}\right)-(1-\varepsilon) \gamma_{0}\right\} \phi(x) .
\end{aligned}
$$

Since, by (2.17),

$$
W_{\lambda}(x, x+z)-W_{\lambda}(x, x-z)=\lambda\left(1+|x+z|^{2}\right)-\lambda\left(1+|x-z|^{2}\right)=4 \lambda\langle x, z\rangle,
$$

we have

$$
\begin{aligned}
\int_{\{0<|z|<1\}}\langle x, z\rangle\left(W_{\lambda}(x, x+z)-W_{\lambda}(x, x-z)\right) \nu(\mathrm{d} z) & =4 \lambda \int_{\{0<|z|<1\}}\langle x, z\rangle^{2} \nu(\mathrm{d} z) \\
& =4 \lambda F(x)|x|^{2},
\end{aligned}
$$

which implies that for any $x \in B\left(0, R_{0}\right)^{c}$,

$$
\begin{aligned}
& \frac{1}{2} \int_{\{0<|z|<1\}}\langle\nabla \phi(x), z\rangle\left(W_{\lambda}(x, x+z)-W_{\lambda}(x, x-z)\right) \nu(\mathrm{d} z) \\
& =-\frac{\delta}{2\left(1+|x|^{2}\right)^{\delta / 2+1}} \int_{\{0<|z|<1\}}\langle x, z\rangle\left(W_{\lambda}(x, x+z)-W_{\lambda}(x, x-z)\right) \nu(\mathrm{d} z) \\
& \leq-2 \lambda \delta(1-\varepsilon) F(x) \phi(x) .
\end{aligned}
$$

The last inequality above holds true due to the fact that we can choose $R_{0}$ large enough 
if necessary. Putting (2.24) and (2.23) into (2.10), we get for any $x \in B\left(0, R_{0}\right)^{c}$

$$
\begin{aligned}
\mathcal{L}_{\lambda} \phi(x) & \leq \lambda \delta\left\{(1+\varepsilon)(\delta+2)\left(F(x)+\frac{c_{1}}{6|x|^{2}}\right)-(1-\varepsilon)\left(\gamma_{0}+2 F(x)\right)\right\} \phi(x) \\
& =\lambda \delta\left\{((1+\varepsilon) \delta+4 \varepsilon) F(x)+\frac{c_{1}(1+\varepsilon)(\delta+2)}{6|x|^{2}}-(1-\varepsilon) \gamma_{0}\right\} \phi(x) \\
& \leq \lambda \delta\left\{((1+\varepsilon) \delta+5 \varepsilon-1) \gamma_{0}+\frac{c_{1}(1+\varepsilon)(\delta+2)}{6|x|^{2}}\right\} \phi(x) \\
& \leq \lambda \delta \gamma_{0}\{(1+\varepsilon) \delta+6 \varepsilon-1\} \phi(x) .
\end{aligned}
$$

Here in the second inequality we used the fact that $F(x) \leq \gamma_{0}$ for any $x \in B\left(0, R_{0}\right)^{c}$, and the last inequality holds true again because we can choose $R_{0}$ large enough if necessary.

For any $\delta \in(0,1)$, we can take $\varepsilon \in(0,1)$ so small that

$$
(1+\varepsilon) \delta+6 \varepsilon-1<0 .
$$

Then, (2.15) follows by letting in (2.25)

$$
\lambda=\frac{C}{\delta \gamma_{0}\{(1-6 \varepsilon)-(1+\varepsilon) \delta\}} .
$$

Hence the proof is complete.

Remark 2.7. As seen from (2.16), (2.22) and (2.25) above, the negativity of $\mathcal{L}_{\lambda} \phi$ is mainly due to the estimate for the term (II). Furthermore, (II) is deduced from the Taylor formula for the test function $\phi$, and roughly speaking it comes from the expansion term $\frac{\partial^{2} \phi}{\partial x_{i} \partial x_{j}}$. This indicates that, for the generator $\mathcal{L}_{\lambda}$ of the quadratic form $\left(\mathcal{E}^{\lambda}, \mathcal{F}\right)$ with finite range jumping kernel, the estimate of $\mathcal{L}_{\lambda} \phi(x)$ for $|x|$ large enough is similar to the second order elliptic operator acting on $\phi$. We also note that, the condition $\delta \in(0,1)$ and the inequality $F(x) \leq \gamma_{0}$ are crucial for the proof of Proposition 2.6.

We also need the following Hardy type inequality for the Dirichlet form $(\mathcal{E}, \mathcal{F})$.

Lemma 2.8. Let $\lambda>0$ and $\phi(x)=\left(1+|x|^{2}\right)^{-\delta / 2}$ for some $\delta>0$. If the function $-\mathcal{L}_{\lambda} \phi / \phi$ is bounded from below, then for any $f \in \mathcal{F}$,

$$
\mathcal{E}(f, f) \geq 2 \int_{\mathbb{R}^{d}} \frac{-\mathcal{L}_{\lambda} \phi}{\phi} f^{2} \mathrm{~d} x .
$$

Proof. We first assume that $f \in C_{c}^{\infty}\left(\mathbb{R}^{d}\right)$. Since $f^{2} / \phi \in C_{c}^{\infty}\left(\mathbb{R}^{d}\right)$, we have by Proposition 2.5(iii),

$$
\begin{aligned}
& \int_{\mathbb{R}^{d}} \frac{-\mathcal{L}_{\lambda} \phi}{\phi}(x) f(x)^{2} \mathrm{~d} x \\
& =\int_{\mathbb{R}^{d}}\left(-\mathcal{L}_{\lambda} \phi\right)(x) \frac{f^{2}}{\phi}(x) \mathrm{d} x=\frac{1}{2} \mathcal{E}^{\lambda}\left(\phi, f^{2} / \phi\right) \\
& =\frac{1}{2} \iint_{\{0<|z|<1\}}(\phi(x+z)-\phi(x))\left(\frac{f^{2}}{\phi}(x+z)-\frac{f^{2}}{\phi}(x)\right) W_{\lambda}(x, x+z) \nu(\mathrm{d} z) \mathrm{d} x .
\end{aligned}
$$


Because for any $x, y \in \mathbb{R}^{d}$,

$$
\begin{aligned}
(\phi(y)-\phi(x))\left(\frac{f^{2}}{\phi}(y)-\frac{f^{2}}{\phi}(x)\right) & =f(y)^{2}-\left(f(x)^{2} \frac{\phi(y)}{\phi(x)}+f(y)^{2} \frac{\phi(x)}{\phi(y)}\right)+f(x)^{2} \\
& \leq f(y)^{2}-2|f(y)||f(x)|+f(x)^{2} \leq(f(y)-f(x))^{2}
\end{aligned}
$$

we have by (2.26) and Assumption 2.3,

$$
\int_{\mathbb{R}^{d}} \frac{-\mathcal{L}_{\lambda} \phi}{\phi}(x) f(x)^{2} \mathrm{~d} x \leq \frac{1}{2} \iint_{\{0<|z|<1\}}(f(x+z)-f(x))^{2} W_{\lambda}(x, x+z) \nu(\mathrm{d} z) \mathrm{d} x \leq \frac{1}{2} \mathcal{E}(f, f) .
$$

We next assume that $f \in \mathcal{F}$. Take a sequence $\left\{f_{n}\right\}_{n \geq 1} \subset C_{c}^{\infty}\left(\mathbb{R}^{d}\right)$ such that $\| f_{n}-$ $f \|_{\mathcal{E}_{1}} \rightarrow 0$ as $n \rightarrow \infty$. Let $\left\{f_{n_{k}}\right\}_{k \geq 1}$ be a subsequence such that $f_{n_{k}} \rightarrow f$, a.e. Since the function $-\mathcal{L}_{\lambda} \phi / \phi$ is bounded from below by assumption, we get by an application of Fatou's lemma,

$$
\mathcal{E}(f, f)=\lim _{k \rightarrow \infty} \mathcal{E}\left(f_{n_{k}}, f_{n_{k}}\right) \geq 2 \liminf _{k \rightarrow \infty} \int_{\mathbb{R}^{d}} \frac{-\mathcal{L}_{\lambda} \phi}{\phi}(x) f_{n_{k}}(x)^{2} \mathrm{~d} x \geq 2 \int_{\mathbb{R}^{d}} \frac{-\mathcal{L}_{\lambda} \phi}{\phi}(x) f(x)^{2} \mathrm{~d} x .
$$

This completes the proof.

Now, we are in a position to present the

Proof of Theorem 2.4. We first note that the Nash-type inequality holds for $(\mathcal{E}, \mathcal{F})$. In fact, let $Z:=\left(Z_{t}\right)_{t \geq 0}$ be a Lévy process on $\mathbb{R}^{d}$ with Lévy measure $\mathbf{1}_{\{0<|z|<1\}} \nu(\mathrm{d} z)$. According to [6, Proposition 4.1] and Assumption 2.3)(ii), the process $Z$ has a transition density function $p(t, x)$ with respect to the Lebesgue measure such that for all $t>0$,

$$
\|p(t, \cdot)\|_{\infty} \leq(2 \pi)^{-d / 2} \int_{\mathbb{R}^{d}} e^{-t \varphi(\xi)} \mathrm{d} \xi \leq \beta_{0}(t)<\infty
$$

This along with [15, Theorem 3.3.15] yields that for any $r>0$ and $f \in C_{c}^{\infty}\left(\mathbb{R}^{d}\right)$,

$$
\int_{\mathbb{R}^{d}} f(x)^{2} \mathrm{~d} x \leq \frac{r}{2} \iint_{\{0<|z|<1\}}(f(x+z)-f(x))^{2} \nu(\mathrm{d} y) \mathrm{d} x+\beta_{0}(r)\left(\int_{\mathbb{R}^{d}}|f(x)| \mathrm{d} x\right)^{2} .
$$

According to Assumption 2.3 , we further know that there is a constant $c_{1}>0$ such that for any $r>0$ and $f \in C_{c}^{\infty}\left(\mathbb{R}^{d}\right)$,

$$
\int_{\mathbb{R}^{d}} f(x)^{2} \mathrm{~d} x \leq c_{1} r \mathcal{E}(f, f)+\beta_{0}(r)\left(\int_{\mathbb{R}^{d}}|f(x)| \mathrm{d} x\right)^{2} .
$$

Replacing $r$ with $r / c_{1}$ in the inequality above, we see that for all $r>0$ and $f \in C_{c}^{\infty}\left(\mathbb{R}^{d}\right)$,

$$
\int_{\mathbb{R}^{d}} f(x)^{2} \mathrm{~d} x \leq r \mathcal{E}(f, f)+\beta_{0}\left(r / c_{1}\right)\left(\int_{\mathbb{R}^{d}}|f(x)| \mathrm{d} x\right)^{2}
$$

In particular, by Assumption 2.3(ii) and [15, Theorem 3.3.14], the associated semigroup $\left(P_{t}\right)_{t \geq 0}$ also has a transition density function with respect to the Lebesgue measure. 
We next establish the essential super Poincaré inequality for $(\mathcal{E}, \mathcal{F})$. Let $\phi(x)=$ $\left(1+|x|^{2}\right)^{-\delta / 2}$ for some fixed $\delta \in(0,1)$. According to Proposition 2.6, for any $C>0$, we can take positive constants $\lambda:=\lambda(C), C_{0}:=C_{0}(C)$ and $R_{0}:=R_{0}(C)$ so that for all $x \in \mathbb{R}^{d}$,

$$
\frac{-\mathcal{L}_{\lambda} \phi}{\phi}(x) \geq C \mathbf{1}_{B\left(0, R_{0}\right)^{c}}(x)-C_{0} \mathbf{1}_{B\left(0, R_{0}\right)}(x) .
$$

This along with Lemma 2.8 yields that for any $f \in C_{c}^{\infty}\left(\mathbb{R}^{d}\right)$,

$$
\begin{aligned}
C \int_{B\left(0, R_{0}\right)^{c}} f(x)^{2} \mathrm{~d} x & \leq \int_{\mathbb{R}^{d}} \frac{-\mathcal{L}_{\lambda} \phi}{\phi}(x) f(x)^{2} \mathrm{~d} x+C_{0} \int_{B\left(0, R_{0}\right)} f(x)^{2} \mathrm{~d} x \\
& \leq \frac{1}{2} \mathcal{E}(f, f)+C_{0} \int_{B\left(0, R_{0}\right)} f(x)^{2} \mathrm{~d} x,
\end{aligned}
$$

whence

$$
\int_{B\left(0, R_{0}\right)^{c}} f(x)^{2} \mathrm{~d} x \leq \frac{1}{2 C} \mathcal{E}(f, f)+\frac{C_{0}}{C} \int_{B\left(0, R_{0}\right)} f(x)^{2} \mathrm{~d} x .
$$

On the other hand, let $\eta:=\eta_{R_{0}} \in C_{c}^{\infty}\left(\mathbb{R}^{d}\right)$ take values in $[0,1]$ so that $\eta=1$ on $B\left(0, R_{0}\right)$ and $\eta=0$ on $B\left(0, R_{0}+1\right)^{c}$. In particular, there exists a constant $c_{2}>0$, independently of $R_{0}$, such that for any $x, z \in \mathbb{R}^{d}$,

$$
|\eta(x+z)-\eta(x)| \leq c_{2}|z| .
$$

Then, by (2.27), we have for any $s>0$ and $f \in C_{c}^{\infty}\left(\mathbb{R}^{d}\right)$,

$$
\begin{aligned}
\int_{B\left(0, R_{0}\right)} f(x)^{2} \mathrm{~d} x \leq & \int_{\mathbb{R}^{d}}(f(x) \eta(x))^{2} \mathrm{~d} x \\
\leq & \frac{s}{2} \iint_{\{0<|z|<1\}}(f(x+z) \eta(x+z)-f(x) \eta(x))^{2} \nu(\mathrm{d} z) \mathrm{d} x \\
& +\beta_{0}(s)\left(\int_{\mathbb{R}^{d}}|f(x) \eta(x)| \mathrm{d} x\right)^{2} \\
\leq & s \iint_{\{0<|z|<1\}}(f(x+z)-f(x))^{2} \nu(\mathrm{d} z) \mathrm{d} x \\
& +s \int_{\mathbb{R}^{d}} f(x)^{2}\left(\int_{\{0<|z|<1\}}(\eta(x+z)-\eta(x))^{2} \nu(\mathrm{d} z)\right) \mathrm{d} x \\
& +\beta_{0}(s)\left(\int_{B\left(0, R_{0}+1\right)}|f(x)| \mathrm{d} x\right)^{2} \\
\leq & c_{3} s \mathcal{E}(f, f)+c_{3} s \int_{\mathbb{R}^{d}} f(x)^{2} \mathrm{~d} x+\beta_{0}(s)\left(\int_{B\left(0, R_{0}+1\right)}|f(x)| \mathrm{d} x\right)^{2},
\end{aligned}
$$

where in the last inequality we used (2.29) and (2.9) to show that

$$
\sup _{x \in \mathbb{R}^{d}} \int_{\{0<|z|<1\}}(\eta(x+z)-\eta(x))^{2} \nu(\mathrm{d} z) \leq c_{3} \int_{\{0<|z|<1\}}|z|^{2} \nu(\mathrm{d} z)<\infty .
$$


Combining (2.30) with (2.28), we know that for any $f \in C_{c}^{\infty}\left(\mathbb{R}^{d}\right)$ and $s>0$,

$$
\begin{aligned}
\int_{\mathbb{R}^{d}} f(x)^{2} \mathrm{~d} x \leq & \left(\frac{1}{2 C}+c_{3} s\right) \mathcal{E}(f, f)+c_{3} s \int_{\mathbb{R}^{d}} f(x)^{2} \mathrm{~d} x \\
& +\beta_{0}(s)\left(\int_{B\left(0, R_{0}+1\right)}|f(x)| \mathrm{d} x\right)^{2}+\frac{C_{0}}{C} \int_{B\left(0, R_{0}\right)} f(x)^{2} \mathrm{~d} x \\
\leq & \left\{\frac{1}{2 C}+c_{3} s\left(1+\frac{C_{0}}{C}\right)\right\} \mathcal{E}(f, f)+c_{3} s\left(1+\frac{C_{0}}{C}\right) \int_{\mathbb{R}^{d}} f(x)^{2} \mathrm{~d} x \\
& +\beta_{0}(s)\left(1+\frac{C_{0}}{C}\right)\left(\sup _{z \in B\left(0, R_{0}+1\right)} \psi(z)^{-1}\right)\left(\int_{\mathbb{R}^{d}}|f(x)| \psi(x) \mathrm{d} x\right)^{2},
\end{aligned}
$$

where $\psi$ is any strictly positive function in $L^{2}\left(\mathbb{R}^{d} ; \mathrm{d} x\right)$. We note here that the constant $c_{3}$ above is independent of $C$, but both $C_{0}:=C_{0}(C)$ and $R_{0}:=R_{0}(C)$ depend on $C$.

Now, for any $r>0$, we first take $C=1 / r$ (i.e., $1 / C=r$ and $C_{0}:=C_{0}(C)=C_{0}(1 / r)$ is fixed), and then choose $s:=s(r)=r /\left(2 c_{3}\left(1+r C_{0}(1 / r)\right)\right)>0$ so that $c_{3} s\left(1+C_{0} / C\right)=r / 2$. Then, for any $f \in C_{c}^{\infty}\left(\mathbb{R}^{d}\right)$ and for any strictly positive function $\psi \in L^{2}\left(\mathbb{R}^{d} ; \mathrm{d} x\right)$,

$$
\begin{aligned}
\int_{\mathbb{R}^{d}} f(x)^{2} \mathrm{~d} x \leq & r \mathcal{E}(f, f)+\frac{r}{2} \int_{\mathbb{R}^{d}} f(x)^{2} \mathrm{~d} x \\
& +\alpha(r)\left(\sup _{z \in B\left(0, R_{0}(1 / r)+1\right)} \psi(z)^{-1}\right)\left(\int_{\mathbb{R}^{d}}|f(x)| \psi(x) \mathrm{d} x\right)^{2},
\end{aligned}
$$

where

$$
\alpha(r):=\beta_{0}(s(r))\left(1+r C_{0}(1 / r)\right) .
$$

This in particular implies that for all $0<r \leq 1$,

$$
\int_{\mathbb{R}^{d}} f(x)^{2} \mathrm{~d} x \leq 2 r \mathcal{E}(f, f)+2 \alpha(r)\left(\sup _{z \in B\left(0, R_{0}(1 / r)+1\right)} \psi(z)^{-1}\right)\left(\int_{\mathbb{R}^{d}}|f(x)| \psi(x) \mathrm{d} x\right)^{2} .
$$

Hence, for all $0<r \leq 2$,

$$
\int_{\mathbb{R}^{d}} f(x)^{2} \mathrm{~d} x \leq r \mathcal{E}(f, f)+2 \alpha(r / 2)\left(\sup _{z \in B\left(0, R_{0}(2 / r)+1\right)} \psi(z)^{-1}\right)\left(\int_{\mathbb{R}^{d}}|f(x)| \psi(x) \mathrm{d} x\right)^{2} .
$$

Therefore, for any $r>0$ and $f \in C_{c}^{\infty}\left(\mathbb{R}^{d}\right)$,

$$
\int_{\mathbb{R}^{d}} f(x)^{2} \mathrm{~d} x \leq r \mathcal{E}(f, f)+\beta(r)\left(\int_{\mathbb{R}^{d}}|f(x)| \psi(x) \mathrm{d} x\right)^{2},
$$

where

$$
\beta(r)=2 \alpha((2 \wedge r) / 2))\left(\sup _{z \in B\left(0, R_{0}(2 /(2 \wedge r)+1)\right.} \psi(z)^{-1}\right)
$$


Note that, in (2.31) we can take any strictly positive and bounded function $\psi \in L^{2}\left(\mathbb{R}^{d} ; \mathrm{d} x\right)$; for example, $\psi(x)=(1+|x|)^{-d-\theta}$ with $\theta>0$ or $\psi(x)=e^{-c|x|^{\theta}}$ with $c, \theta>0$. In particular, with this choice, $\beta(r)<\infty$ for all $r>0$. Since $C_{c}^{\infty}\left(\mathbb{R}^{d}\right)$ is $\|\cdot\|_{\mathcal{E}_{1}}$-dense in $\mathcal{F}$, (2.31) is valid for any $f \in \mathcal{F}$. Hence, we obtain the essential super Poincaré inequality for $(\mathcal{E}, \mathcal{F})$. Combining this with [15, Theorems 3.1.7, 3.2.1 and 0.3.9], we prove that the semigroup $\left(P_{t}\right)_{t \geq 0}$ is compact on $L^{2}\left(\mathbb{R}^{d} ; \mathrm{d} x\right)$, also thanks to the assertion claimed above that $\left(P_{t}\right)_{t \geq 0}$ has a transition density function with respect to the Lebesgue measure.

Remark 2.9. As seen from the proof above, Proposition 2.6 is the key ingredient to yield Theorem [2.4. This along with Remark 2.7 partly explain the reason why the criteria for compactness of the semigroup associated with the non-local Dirichlet form $\left(\mathcal{E}^{*}, \mathcal{F}^{*}\right)$ on $L^{2}\left(\mathbb{R}^{d} ; \mathrm{d} x\right)$ with finite range jumping kernel is similar to these for the local Dirichlet form given by (1.1). On the other hand, following the proof of Theorem 2.4 (in particular the comparison argument), one may show that the semigroup associated with the local Dirichlet form $\left(\mathcal{E}^{L}, \mathcal{F}^{L}\right)$ given in Remark 2.2 is compact, if

$$
\liminf _{|x| \rightarrow \infty} \inf _{\xi \in \mathbb{R}^{d}} \frac{\sum_{i, j=1}^{d} a_{i j}(x) \xi_{i} \xi_{j}}{|x|^{2}|\xi|^{2}}=\infty
$$

\section{Proof of Theorem 1.1 and further examples}

We first present the

Proof of Theorem 1.1. Let $W(x, y)$ be as in (1.6) such that $U_{i}(i=1,2)$ is locally bounded and (1.7) holds. Then it is easy to verify the condition (2.3).

We first prove (i). Under the setting in this assertion, $W(x, y)$ satisfies Assumption 2.3(i). Let $J(x, \mathrm{~d} y)=|x-y|^{-d-\alpha} \mathrm{d} y$ and $\nu(\mathrm{d} z)=\mathbf{1}_{\{0<|z|<1\}}|z|^{-d-\alpha} \mathrm{d} z$. Then, the measure $\nu$ is symmetric, and $J(x, x+A)=\nu(A)$ for any $x \in \mathbb{R}^{d}$ and for any Borel set $A \subset B(0,1)$. Moreover, by the proof of [1, Proposition 2.2], there exists $c_{1}>0$ such that for any $\xi \in \mathbb{R}^{d}$,

$$
\int_{\{0<|z|<1\}}(1-\cos \langle z, \xi\rangle) \nu(\mathrm{d} z) \geq c_{1}\left(|\xi|^{2} \wedge|\xi|^{\alpha}\right) .
$$

Hence Assumption 2.3(ii) is fulfilled with $\beta_{0}(r)=c_{2}\left(r^{-d / \alpha} \vee r^{-d / 2}\right)$ for all $r>0$. Then by Theorem 2.4, the semigroup $\left(P_{t}\right)_{t>0}$ is compact on $L^{2}\left(\mathbb{R}^{d} ; \mathrm{d} x\right)$.

We next prove (ii). Under the setting in this assertion, by some simple calculations, we find that for all $l \geq 1$,

$$
\int_{\{|x| \leq l\}} \int_{\mathbb{R}^{d}}\left(1 \wedge \frac{|x-y|^{2}}{l^{2}}\right) W(x, y) J(x, \mathrm{~d} y) \mathrm{d} x \leq c_{3}\left(l^{d}+l^{d+p-\alpha}\right) .
$$

This along with the assumption $p \in[0, \alpha)$ yields that (2.4) holds true. Therefore, the semigroup $\left(P_{t}\right)_{t \geq 0}$ is not compact on $L^{2}\left(\mathbb{R}^{d} ; \mathrm{d} x\right)$ by Theorem 2.1 .

We next present further examples of non-local Dirichlet forms to which Theorems 2.1 and 2.4 are applicable. 
Let $\sigma(d \theta)$ be a finite and symmetric nonnegative measure on the unit sphere $\mathbb{S}$ in $\mathbb{R}^{d}$. Assume that $\sigma$ is nondegenerate in the sense that its support is not contained in any proper linear subspace of $\mathbb{R}^{d}$. For any $\alpha \in(0,2)$, let

$$
\nu(A)=\int_{0}^{1}\left(\int_{\mathbb{S}} \mathbf{1}_{A}(r \theta) \sigma(\mathrm{d} \theta)\right) r^{1+\alpha} \mathrm{d} r, \quad A \in \mathcal{B}\left(\mathbb{R}^{d}\right) .
$$

Then, by the proof of [10, Example 1.5], we know that (3.1) still holds true. With this fact at hand, we have the following two examples, which indicate that Theorems 2.1 and 2.4 work for some degenerate or singular (with respect to the Lebesgue measure) jumping kernels.

Example 3.1. Let $\Lambda$ be an infinite cone on $\mathbb{R}^{d}$ with $d \geq 2$ that has non-empty interior and is symmetric with respect to the origin, i.e., $\lambda x \in \Lambda$ if $x \in \Lambda$ and $\lambda \in \mathbb{R}$. For $\alpha \in(0,2)$, let

$$
\nu(\mathrm{d} z)=\mathbf{1}_{\{0<|z|<1\}} \frac{1}{|z|^{d+\alpha}} \mathbf{1}_{\Lambda}(z) \mathrm{d} z
$$

and $J_{1}(x, \mathrm{~d} y)=\nu(\mathrm{d}(y-x))$. Then, $J_{1}(x, \mathrm{~d} y)$ satisfies Assumption 2.3(ii) with the measure $\nu$. On the other hand, (3.2) holds with $\sigma(\mathrm{d} \theta)=\mathbf{1}_{\Lambda \cap \mathbb{S}}(\theta) \mu(\mathrm{d} \theta)$, where $\mu$ is the Lebesgue surface measure on $\mathbb{S}$. Hence, (3.1) remains valid for $\nu$. Therefore, according to the proof of Theorem 1.1, the assertion of Theorem 1.1 is true with $J(x, \mathrm{~d} y)=|x-y|^{-d-\alpha} \mathrm{d} y$ replaced by the jumping kernel $J_{1}(x, \mathrm{~d} y)$ above.

In fact, following the proof of Theorem 1.1, we can obtain that Theorem 1.1 also holds true for

$$
J_{2}(x, \mathrm{~d} y)=\left(\sum_{n=0}^{\infty}|x-y|^{-(d+\alpha)} \mathbf{1}_{\left\{|x-y| \in\left[2^{-(2 n+1)}, 2^{-2 n}\right)\right\}}\right) \mathrm{d} y,
$$

with $\alpha \in(0,2)$.

Example 3.2. For any $\alpha \in(0,2)$, let

$$
\nu(\mathrm{d} z)=\sum_{i=1}^{d}\left[\mathbf{1}_{\left\{0<\left|z_{i}\right|<1\right\}} \frac{\mathrm{d} z_{i}}{\left|z_{i}\right|^{1+\alpha}} \otimes \prod_{j \neq i} \delta_{0}\left(\mathrm{~d} z_{j}\right)\right],
$$

where $\delta_{0}$ is the Dirac measure at $0 \in \mathbb{R}$. Let $\left\{e_{i}\right\}_{i=1}^{d}$ be the standard orthonormal basis of $\mathbb{R}^{d}$ and

$$
\sigma(\mathrm{d} \theta):=\sum_{i=1}^{d} \delta_{ \pm e_{i}}(\mathrm{~d} \theta)
$$

where $\delta_{\theta_{0}}(\mathrm{~d} \theta)$ denotes the Dirac measure on $\mathbb{S}$ concentrated at $\theta_{0} \in \mathbb{S}$. Then the measure $\nu$ satisfies (3.1) because (3.2) holds with this $\sigma$. Therefore, by the proof of Theorem 1.1, the assertion of Theorem 1.1 is true with $J(x, \mathrm{~d} y)=|x-y|^{-d-\alpha} \mathrm{d} y$ replaced by the jumping kernel $J_{3}(x, \mathrm{~d} y)=\nu(\mathrm{d}(y-x))$.

Indeed, we can further extend the example above to more general setting. Let $d=$ $d_{1}+d_{2}$ with $d_{i} \geq 1$ for $1 \leq i \leq 2$. For $i=1,2$, let $\delta_{z}^{(i)}$ be the $d_{i}$-dimensional Dirac measure 
at $z \in \mathbb{R}^{d_{i}}$, and $m^{(i)}$ the $d_{i}$-dimensional Lebesgue measure. For $\alpha_{1}, \alpha_{2} \in(0,2)$, define a rotationally invariant measure $\nu$ on $\mathbb{R}^{d_{1}+d_{2}}$ by

$$
\begin{aligned}
\nu(\mathrm{d} z)= & \nu\left(\mathrm{d} z_{1} \times \mathrm{d} z_{2}\right) \\
= & \mathbf{1}_{\left\{0<\left|z_{1}\right|<1\right\}} \frac{1}{\left|z_{1}\right|^{d_{1}+\alpha_{1}}} m^{(1)}\left(\mathrm{d} z_{1}\right) \otimes \delta_{0}^{(2)}\left(\mathrm{d} z_{2}\right) \\
& +\mathbf{1}_{\left\{0<\left|z_{2}\right|<1\right\}} \frac{1}{\left|z_{2}\right|^{d_{2}+\alpha_{2}}} \delta_{0}^{(1)}\left(\mathrm{d} z_{1}\right) \otimes m^{(2)}\left(\mathrm{d} z_{2}\right),
\end{aligned}
$$

and the kernel $J_{4}(x, \mathrm{~d} y)$ on $\mathbb{R}^{d_{1}+d_{2}} \times \mathcal{B}\left(\mathbb{R}^{d_{1}+d_{2}}\right)$ by

$$
\begin{aligned}
J_{4}(x, \mathrm{~d} y)= & \nu(\mathrm{d}(y-x)) \\
= & \mathbf{1}_{\left\{0<\left|x_{1}-y_{1}\right|<1\right\}} \frac{1}{\left|x_{1}-y_{1}\right| d_{1}+\alpha_{1}} m^{(1)}\left(\mathrm{d} y_{1}\right) \delta_{x_{2}}^{(2)}\left(\mathrm{d} y_{2}\right) \\
& +\mathbf{1}_{\left\{0<\left|x_{2}-y_{2}\right|<1\right\}} \frac{1}{\left|x_{2}-y_{2}\right|^{d_{2}+\alpha_{2}}} \delta_{x_{1}}^{(1)}\left(\mathrm{d} y_{1}\right) m^{(2)}\left(\mathrm{d} y_{2}\right) .
\end{aligned}
$$

Note that the measure $\nu(\mathrm{d} z)$ above is a Lévy measure of the direct product of truncated symmetric $\alpha_{i}$-stable processes on $\mathbb{R}^{d_{i}}$ with $i=1,2$. Then, we can claim that the assertion of Theorem 1.1 remains true with $q \in\left[0, \alpha_{1} \wedge \alpha_{2}\right)$ and the jumping kernel $J_{4}(x, \mathrm{~d} y)$ above.

Acknowledgements. The research of Yuichi Shiozawa is supported in part by JSPS KAKENHI No. JP17K05299. The research of Jian Wang is supported by the National Natural Science Foundation of China (No. 11831014), the Program for Probability and Statistics: Theory and Application (No. IRTL1704), and the Program for Innovative Research Team in Science and Technology in Fujian Province University (IRTSTFJ).

\section{References}

[1] Z.-Q. Chen, P. Kim and T. Kumagai, Weighted Poincaré inequality and heat kernel estimates for finite range jump processes, Math. Ann., 342 (2008), 833-883.

[2] Z.-Q. Chen and T. Kumagai, Heat kernel estimates for stable-like processes on $d$-sets, Stochastic Process. Appl., 108 (2003), 27-62.

[3] E. B. Davies, $L^{1}$ properties of second order elliptic operators, Bull. London Math. Soc., 17 (1985), 417-436.

[4] E. B. Davies, Heat Kernels and Spectral Theory, Cambridge University Press, Cambridge, 1990.

[5] M. Fukushima, Y. Oshima and M. Takeda, Dirichlet Forms and Symmetric Markov Processes, 2nd rev. and ext. ed., Walter de Gruyter, 2011.

[6] V. Knopova and R. L. Schilling, A note on the existence of transition probability densities of Lévy processes, Forum Math., 25 (2013), 125-149. 
[7] K. Matsuura, Compactness of semigroups of explosive symmetric Markov processes, to appear in Kyoto J. Math., available at arXiv:1808.01799.

[8] M. M. H. Pang, $L^{1}$ properties of two classes of singular second order elliptic operators, J. London. Math. Soc., 38 (1988), 525-543.

[9] M. M. H. Pang, $L^{1}$ and $L^{2}$ properties of a class of singular second order elliptic operators on $\mathbb{R}^{N}$ with measurable coefficients, J. Differential Equations, 129 (1996), $1-17$.

[10] R. L. Schilling, P. Sztonyk and J. Wang, Coupling property and gradient estimates for Lévy processes via the symbol, Bernoulli, 18 (2012), 1128-1149.

[11] Y. Shiozawa and T. Uemura, Explosion of jump-type symmetric Dirichlet forms on $\mathbb{R}^{d}$, J. Theoret. Probab., 27 (2014), 404-432.

[12] M. Takeda, Compactness of symmetric Markov semigroups and boundedness of eigenfunctions, Trans. Amer. Math. Soc., 372 (2019), 3905-3920.

[13] M. Takeda, Y. Tawara and K. Tsuchida, Compactness of Markov and Schrödinger semi-groups: A probabilistic approach, Osaka J. Math., 54 (2017), 517-532.

[14] F.-Y. Wang, Functional inequalities and spectrum estimates: the infinite measure case, J. Funct. Anal., 194 (2002), 288-310.

[15] F.-Y. Wang, Functional Inequalities, Markov Processes and Spectral Theory, Science Press, Beijing (2005).

[16] J. Wang, Compactness and density estimates for weighted fractional heat semigroups, J. Theoret. Probab., 32 (2019), 2066-2087.

[17] L. Wu, Uniformly integrable operators and large deviations for Markov processes, J. Funct. Anal., 172 (2000), 301-376. 